\title{
Butyrate Supplementation Affects Mrna Abundance of Genes Involved in Glycolysis, Oxidative Phosphorylation and Lipogenesis in the Rumen Epithelium of Holstein Dairy Cows
}

\author{
${ }^{1}$ Anne Hermen Laarman, ${ }^{1}$ Louis Dionissopoulos, \\ ${ }^{1}$ Ousama AIZahal, ${ }^{1}$ Michael Alexander Steele, \\ ${ }^{2}$ Sabrina Louise Greenwood, ${ }^{3}$ James Clyde Matthews and ${ }^{1}$ Brian William McBride \\ ${ }^{1}$ Department of Animal and Poultry Science, \\ Ontario Agricultural College, University of Guelph, Guelph ON, Canada \\ ${ }^{2}$ Department of Animal Science, \\ College of Agriculture and Life Sciences, University of Vermont, Burlington, VT, USA \\ ${ }^{3}$ Department of Animal and Food Sciences, \\ College of Agriculture, University of Kentucky, Lexington, KY, USA
}

Received 2013-10-28; Revised 2013-11-21; Accepted 2013-11-27

\begin{abstract}
Energy availability in epithelial cells is a crucial link for maintaining epithelial barrier integrity; energy depletion is linked to impaired barrier function in several epithelia. This study aimed to elucidate the effects of exogenous butyrate on mRNA abundance of genes indirectly involved in rumen epithelial barrier integrity. Sixteen mid-lactation Holstein cows fed a total mixed ration received a concentrate mix to induce Subacute Ruminal Acidosis (SARA). For 7 days, while being fed the concentrate mix, cows were assigned either a control treatment or a butyrate treatment, in which cows were fed butyrate at $2.5 \%$ daily dry matter intake in the form of a calcium salt. On days 6 and 7, rumen $\mathrm{pH}$ was measured continuously and on day 7, rumen biopsies took place. Rumen $\mathrm{pH}$ fell below 5.6 for more than 3 hours per day in both treatments, con-firming the occurrence of SARA. Microarray and pathway analysis, confirmed by real time PCR, showed that exogenous butyrate significantly increased the mRNA abundance of hexokinase 2 (fold change: 2.07), pyruvate kinase (1.19), cytochrome B-complex 3 (1.18) and ATP Synthase, F0 subunit (1.66), which en-code important glycolytic enzymes. Meanwhile, butyrate decreased mRNA abundance of pyruvate dehydrogenase kinase 2(-2.38), ATP citrate lyase (-2.00) and mitochondrial CoA transporter (-2.27), which en-code enzymes involved in lipogenesis. These data suggest exogenous butyrate induces a shift towards energy mobilization in the rumen epithelium, which may aid barrier function in the rumen epithelium during SARA.
\end{abstract}

Keywords: Rumen Epithelium, Butyrate, Energy Mobilization

\section{INTRODUCTION}

In cows fed a high grain diet for milk production purposes, Subacute Ruminal Acidosis (SARA) continues to be a costly problem, with one estimate of $\$ 400$ USD in lost milk production per cow per lactation (Plaizier et al., 2008). The accumulation of Short Chain Fatty Acids (SCFA) leads to SARA and strains the integrity of the rumen epithelium (Zebeli and MetzlerZebeli, 2012). In cases where SARA compromises epithelial integrity, many detrimental health effects can result, such as liver abscesses, lesions and even University of Guelph, Guelph ON, Canada 
laminitis (Krause and Oetzel, 2006). Adverse health events are caused by translocation of bacteria across the rumen epithelium, underscoring the importance of epithelial integrity.

Like other epithelia, ruminal barrier function is attained by tight junction proteins such as tight junction protein isoform 3 , zonal occludins isoform 1 andclaudin isoform 1. These proteins physically anchor the cells of the stratum granulosum (Graham and Simmons, 2005), effectively eliminating paracellular bacterial translocation. In so doing, the epithelium can facilitate SCFA and proton transport across the rumen epithelium using multiple transport mechanisms, in addition to a smaller fraction of SCFA that passively diffuses across the epithelium (Aschenbach et al., 2009). Maintaining epithelial integrity thus plays a crucial role in health maintenance in dairy cows.

One possible nutraceutical implicated in maintaining barrier function is butyrate, which is shown to genomically regulate several cellular processes and modulate gene expression through Histone Deacetylase Complex (HDAC) inhibition (Ploger et al., 2012). In cows, butyrate increases expression of critical barrier function genes, including tight junction proteins and claudins (Baldwin et al., 2012). Another example is in Caco-2 cells, where butyrate decreases epithelial permeability and increases transepithelial resistance at low concentrations (Peng et al., 2007). On a protein level, butyrate promotes redistribution of tight junction proteins through activation of AMP-Activated Protein Kinase (AMPK) (Peng et al., 2009). Activity of AMPK is required for maintaining epithelial cell polarity, but only in a low energetic state, highlighting the importance of energy state in epithelial function (Mirouse et al., 2007). In freshly weaned piglets, low energy state adversely affects barrier function in the small intestine, whereas varying dietary inclusions of protein and lactose do not (Spreeuwenberg et al., 2001). How butyrate might affect energy status in epithelial cells is unclear.

The objective of this study was to determine how butyrate affects genes involved with energy mobilization in the rumen epithelium. Butyrate's importance in the homeostasis of many processes can clearly be seen in previous studies. Whether genes involved in energy status dynamics are affected by butyrate, however, is unclear. We therefore hypothesized that butyrate would alter gene expression for the purpose of energy mobilization in the rumen epithelium to prevent energetic stress caused by SARA.

\section{MATERIALS AND METHODS}

\subsection{Animals, Treatment and Sampling}

This study was carried out as described previously (Dionissopoulos et al., 2013). All animal procedures were approved by the Animal Care Committee at the University of Guelph under the guidelines of the Canadian Council for Animal Care (Ottawa, ON, CA). Sixteen fistulated cows on a mid-lactation Total Mixed Ration (TMR) were fed a grain supplement that increased dietary Non-Fibre Carbohydrate (NFC) content to $44.0 \%$ on a dry matter basis. Two days before the start of the study, only half the supplement was fed and on the day before the study, the full amount of supplement was fed. Thereafter, cows were divided into a control treatment and a butyrate treatment. Cows on the control treatment received a carrier only, while cows on the butyrate treatment received a carrier containing a butyrate dose (Proformix; Probiotech Inc., Saint Hyacinthe, QC) at the rate of $2.5 \%$ of Dry Matter Intake (DMI). Daily, DMI and milk production were measured. On days 1 and 7, blood was sampled and analyzed for serum $\beta$-Hydroxybutyrate (BHBA) by the Animal Health Laboratory (Guelph, ON, Canada) using established protocols (Williamson et al., 1962). Also on the same day, rumen fluid from the ventral sac of the rumen was strained through 4 layers of cheesecloth and analyzed for SCFA profile by gas chromatography as described previously (Mutsvangwa et al., 2002). On days 6 and 7, rumen $\mathrm{pH}$ was continuously measured using an indwelling pH monitoring system (AlZahal et al., 2007). Rumen biopsies were done on day 7 by partial evacuation of the rumen and processed for microarray and quantitative Real-Time PCR (qRT-PCR).

\subsection{Microarray and $q R T-P C R$}

Rumen papillae samples were analyzed by microarray as previously described (Dionissopoulos et al., 2013). Samples from the control treatment were pooled and the butyrate samples were compared to the pooled control samples using student's t-test (Xue et al., 2010). Significance was determined using a pre-screen of $95 \%$ confidence and a false discovery rate of 0.1 . Through Ingenuity Pathway Analysis (Ingenuity Inc., Redwood City, CA, USA), networks of interconnected differentially expressed genes were created and a subset of differentially expressed genes were identified for confirmation by PCR. Primers were designed using Primer-BLAST (NCBI, Bethesda, MD, USA); most primers were intron-spanning (Table 1). 
Table 1. Primer sequences for genes verified by PCR

\begin{tabular}{|c|c|c|c|c|}
\hline Gene & Name & Accession No. & Primer sequence $(\mathrm{F} / \mathrm{P})$ & $\mathrm{E}(\%)$ \\
\hline \multirow[t]{2}{*}{ HK2 } & Hexokinase 2 & XM_002691189.2 & F AGTGCAGAAGGTTGACCAGT & 106 \\
\hline & & & P CCAAAGCACACGGAAGTTGG & \\
\hline \multirow[t]{2}{*}{ PKM2 } & Pyruvate kinase, muscle & ВT030503.1 & F GCCATGAATGTCGGAAAGGC & 93 \\
\hline & & & P GATGGTTTGGGGAAGAGGGG & \\
\hline \multirow[t]{2}{*}{ LDHA } & Lactate dehydrogenase A & NM_174099 & F GTCAGCAGTCTGGCAGCTAT & 101 \\
\hline & & & P TAACCAGCCTGGAGTTTGCT & \\
\hline \multirow[t]{2}{*}{ LDHB } & Lactate dehydrogenase B & NM_174100 & F TCGTGCAGCCCTTATCACTC & 85 \\
\hline & & & P CGTCAGTCAGAGACTTTCCCA & \\
\hline \multirow[t]{3}{*}{ PFKFB4 } & 6-phosphofructo-2-kinase/fructose & NM_001192835 & F ATGACCAACTGTCCAACGCT & \\
\hline & -2,6-biphosphatase 4 & & ATGACCAACTGTCCAACGCT & 99 \\
\hline & & & P TGTTGCATCAAAAACCGCCA & \\
\hline \multirow[t]{2}{*}{ ACAD9 } & Acyl-CoA dehydrogenase 9 & NM_001078076 & F GTCTGGGTCACCAATGGAGG & 93 \\
\hline & & & P TGACGCCACCAAAGTCTCTC & \\
\hline \multirow[t]{2}{*}{ CYBASC3 } & Cytochrome B561, member A3 & NM_001099149 & F TGTTGCCGAGAGTCTGTCAC & 83 \\
\hline & & & P CTGACCAGGCAGCCCTTTAT & \\
\hline \multirow[t]{2}{*}{ ATP5G1 } & ATP synthase, $F_{0}$ subunit & NM_176649 & F CTATGCCAGGAACCCGTCTC & 88 \\
\hline & & & P AGGTTAGCACACTCCAGCAC & \\
\hline \multirow{2}{*}{ ACLY } & ATP citrate lyase & NM_001037457 & F TTGGAGAGATAGGGGGCACA & 94 \\
\hline & & & P TGGACCTCGGAGGAGAACAT & \\
\hline \multirow[t]{2}{*}{ FASN } & Fatty acid synthase & NM_001012669 & F CTTCCAGTGGGTTGACTCCC & 90 \\
\hline & & & P CTCCTCGGGCTTGTCTTGTT & \\
\hline \multirow[t]{2}{*}{ PDK2 } & Pyruvate dehydrogenase kinase 2 & NM_001159481 & F CCCGAGTCCTAGAAGTGGTC & 93 \\
\hline & & & P GGACATACCAGCTCTGTACCA & \\
\hline \multirow[t]{2}{*}{ SLC25A42 } & Mitochondrial CoA transporter & NM_001192032 & F GCACGTCTCCTCAAAGAGTG & 80 \\
\hline & & & P GCACGTCTCCTCAAAGAGTG & \\
\hline \multirow[t]{2}{*}{ ATP1B1 } & $\mathrm{Na} / \mathrm{K}$ ATPase & NM_001035334 & F GCCCCACCAGGATTAACACA & 96 \\
\hline & & & P TGGGATCGTTAGGACGAAAGG & \\
\hline
\end{tabular}

Primers were analyzed by Basic Local Alignment Search Tool (NCBI, Bethesda, MD, USA) to confirm specificity to the target gene and to confirm a low risk of nonspecific binding. Primers were then further analyzed by OligoAna-lyzer (Integrated DNA Technologies, Coralville, IA, USA) for primer-primer heterodimers, self-dimers and hairpins. After ordering the primers (Sigma-Aldrich, Oakville, ON, Canada), primer fidelity was empirically confirmed through dissociation curves. GAPDH was used as an internal control gene for quantification using previous methods (Pfaffl, 2001).

\section{RESULTS}

\subsection{SCFA, Blood, Rumen pH}

Total SCFA concentrations were higher on day 1 in the butyrate treatment $(92.76 \pm 4.51 \mathrm{Vs} 78.87 \pm 4.51 \mathrm{mM})$ but not on day $7(87.59 \pm 4.51 \mathrm{Vs} 81.82 \pm 4.51 \mathrm{mM})$. Butyrate concentrations on both days 1 and 7 were higher in butyrate than in control $(22.60 \pm 0.94 \mathrm{Vs} 9.88 \pm 0.94 \mathrm{mM}$ and 21.60 $\pm 0.94 \mathrm{Vs} 8.60 \pm 0.94 \mathrm{mM}$; Dionissopoulos et al., 2013). Serum BHBA was higher in the butyrate treatment than the control treatment on both days 1 (4201 $\pm 265 \mathrm{Vs}$
$910 \pm 265 \mu \mathrm{M})$ and $7 \quad(3262 \pm 265 \quad$ Vs $800 \pm 265 \mu \mathrm{M}$; Dionissopoulos et al., 2013). Rumen $\mathrm{pH}$ measurements confirmed the occurrence of acidosis in both butyrate and control treatments, as indicated by the time rumen $\mathrm{pH}$ was less than 5.6 (536 \pm 89 and $598 \pm 97 \mathrm{~min} /$ day, respectively; Dionissopoulos et al., 2013).

\subsection{Microarray and $q R T-P C R$}

Pathway analysis showed arrays of glycolysis, lipogenesis and oxidative phosphorylation genes as being differentially expressed. Of the 31 genes identified by pathway analysis, 13 genes in these 3 processes were confirmed by qRT-PCR (Table 2). In cows on the butyrate treatment, glycolytic genes such as hexokinase 2 (fold change: 2.07), pyruvate kinase 2 (FC:1.19), lactate dehydrogenase A (FC:1.45), lactate dehydrogenase B $(\mathrm{FC}: 1.18)$ were upregulated while the phosphofructokinase inhibitor 6-phosphofructo-2kinase/fructose-2,6-biphosphatase 4 (FC:-2.22) was downregulated. Criticaloxidative phosphorylation genes were upregulated, including acyl-CoA dehydrogenase 9 (FC:-1.30), cytochrome B561, member A3 (FC: 1.11) and ATP synthase F0 subunit (FC: 1.66). 
Anne Hermen Laarman et al. / American Journal of Animal and Veterinary Sciences 8 (4): 239-245, 2013

Table 2. Microarray and quantitative RT-PCR analysis of genes differentially expressed by microarray

\begin{tabular}{|c|c|c|c|c|}
\hline Gene & Name & Role & $\begin{array}{l}\text { Microarray } \\
\text { fold change }\end{array}$ & $\begin{array}{l}\text { PCR Fold } \\
\text { change }\end{array}$ \\
\hline$\overline{\mathrm{HK} 2}$ & Hexokinase 2 & Glycolysis & 1.51 & 2.07 \\
\hline PKM2 & Pyruvate kinase, muscle & Glycolysis & 1.34 & 1.19 \\
\hline LDHA & Lactate dehydrogenase A & Glycolysis & 1.33 & 1.45 \\
\hline LDHB & Lactate dehydrogenase B & Glycolysis & 1.23 & 1.18 \\
\hline PFKFB4 & $\begin{array}{l}\text { 6-phosphofructo-2-kinase/fructose- } \\
\text { 2,6-biphosphatase } 4\end{array}$ & Glycolysis & -1.37 & -2.22 \\
\hline GPI & Glucose-6-phosphate isomerase & Glycolysis & 1.18 & N/A \\
\hline PFKL & Phosphofructokinase, liver & Glycolysis & 1.18 & N/A \\
\hline ACAD9 & Acyl-CoA dehydrogenase 9 & Oxidative phosphorylation & 1.18 & -1.30 \\
\hline CYBASC 3 & Cytochrome B561, member A3 & Oxidative phosphorylation & 1.18 & 1.11 \\
\hline ATP5G1 & ATP synthase, $F_{0}$ subunit & Oxidative phosphorylation & 1.15 & 1.66 \\
\hline ACLY & ATP citrate lyase & Lipogenesis & -1.31 & -2.00 \\
\hline FASN & Fatty acid synthase & Lipogenesis & 1.27 & 1.48 \\
\hline PDK2 & Pyruvate dehydrogenase kinase 2 & Lipogenesis & -1.37 & -2.38 \\
\hline SLC25A42 & Mitochondrial CoA transporter & Lipogenesis & -1.39 & -2.27 \\
\hline ACSS2 & Acyl-CoA synthetase short chain & Lipogenesis & -1.47 & N/A \\
\hline SCD & Stearoyl-CoA desaturase (delta9) & Lipogenesis & 1.35 & $\mathrm{~N} / \mathrm{A}$ \\
\hline SLC27A2 & Long chain fatty acid transporter & Lipogenesis & -2.21 & $\mathrm{~N} / \mathrm{A}$ \\
\hline ATP1B1 & $\mathrm{Na} / \mathrm{K}$ ATPase & Metabolic activity & 1.75 & 1.50 \\
\hline
\end{tabular}

Also, genes involved in lipogenesis were mostly downregulated, including ATP citrate lyase (FC: -2.00), fatty acid synthase (FC: 1.48), pyruvate dehydrogenase kinase 2 (FC: -2.38 ) and mitochondrial CoA transporter (FC: -2.27). Also, sodium/potassium ATPase was upregulated (FC: 1.50 ) by butyrate treatment.

\section{DISCUSSION}

\subsection{Metabolic Stress and Barrier Integrity}

Energy demands in epithelial tissues can be quite high and potentially very indicative of epithelial barrier integrity status. Infection by rotavirus of Caco2 cells causes concurrent drops in transepithelial resistance and epithelial ATP concentrations (Dickman et al., 2000). In rumen epithelia, low mucosal $\mathrm{pH}$ and local ATP depletion are directly linked to increased epithelial permeability (Aschenbach et al., 2000). Thus, generating adequate ATP in the epithelium is vital for the maintenance of epithelial integrity. Increased sodium/potassium ATPase mRNA in the butyrate treatment highlights a genomic shift to increased ATP demand. In previous research (McBride and Kelly, 1990), increased sodium/potassium ATPase (ATP1B1) activity was directly associated with increased ATP demand in rumen epithelium. Together, these studies highlight the vital role of ATP in maintaining epithelial integrity.
Energy substrate availability and ATP generation capacity in epithelial tissues are vital for maintaining ATP levels. For example, impairment of glucose transport dynamics through the glucose transporter GLUT1, causes significant impairment of blood-brain barrier function by reducing the expression of occludin in brain microvessels (Muneer et al., 2011). In rat pulmonary epithelium, impaired mitochondrial complex I function leads to ATP-depletion and increased endothelial permeability in lung tissue (Bongard et al., 2013). In T84 cell lines, inhibition of ATP synthesis through 2,4dinitrophenol, coupled with $E$. coli exposure, significantly reduces barrier function (Lewis and McKay, 2009). Since the bovine rumen typically has high $E$. coli content, decreased electron transport chain function would likely have an adverse effect on barrier function. In summary, energy substrates must be abundant and metabolizable to ATP for maintenance of barrier function. Impairing the barrier integrity appears to be a direct function of ATP depletion, highlighting the importance of ATP levels in maintaining barrier integrity.

\subsection{Butyrate and Metabolic Stress}

Stressed epithelia, such as those in ulcerative colitis and Crohn's disease, are positively impacted by butyrate (Leonel and Alvarez-Leite, 2012; Ploger et al., 2012), through several possible cellular mechanisms, such as HDAC inhibition (Davie, 2003). A recent study, 
however, suggests butyrate acts as an energy substrate rather than an HDAC inhibitor (Donohoe et al., 2011). This may be due to butyrate's extensive metabolism in the epithelium, where $75-90 \%$ of absorbed butyrate is metabolized (Bergman, 1990). When mouse embryonic stem cells are exposed to sodium butyrate, however, glycolysis and glucose consumption rates increase (Sharma et al., 2006). While butyrate oxidation certainly provides energy to epithelial cells (Bergman, 1990), this energy is made available through its oxidation via the Kreb's Cycle, not through glycolysis. Although increased use of butyrate as an energy substrate cannot be ruled out by our results, the mRNA abundance increases in glycolytic genes in our study, show butyrate acts at the genome level.

Genomically, butyrate inhibits HDAC, resulting in lower deacetylation of lysine residues of histone proteins. Normally, deacytelation of histone proteins is an important and quick-acting buffer of intracellular $\mathrm{pH}$ because the released acetate drives proton export through monocarboxylate co-transporters (McBrian et al., 2013). With inhibition of HDAC, this source of acetate is unavailable and intracellular $\mathrm{pH}$ can drop accordingly. Changes in intracellular $\mathrm{pH}$ have pleiotropic consequences, from changes in ion transport, to changes in cell proliferation, to apoptosis (Casey et al., 2010), highlighting the importance of HDAC activity.

In glioma cells, HDAC inhibition by butyrate enhances apoptosis, but only when glycolysis is also blocked (Egler et al., 2008). Glycolysis, therefore, appears to be a crucial compensatory mechanism for HDAC inhibition. In mice, butyrate decreased HDAC activity and caused a concurrent increase in systemic energy expenditure (Gao et al., 2009). With increased systemic energy expenditures comes an increased energy demand, which could be filled by ATP-generating processes like glycolysis. In our study, butyrate increased relative mRNA abundance of hexokinase 2 , pyruvate kinase 2, lactate dehydrogenases $\mathrm{A}$ and $\mathrm{B}$ and decreased mRNA expression of 6-phosphofructo-2kinase/fructose-2,6-biphosphatase $\quad 4, \quad$ a phosphofructokinase inhibitor. Together, this suggests butyrate increases mRNA abundance of glycolysis genes, which may lead to a shift towards greater glycolytic activity in the rumen epithelium.

Other than glycolysis, oxidative phosphorylation is another cellular ATP-generator and a far more effective one. Oxidative phosphorylation is increased by butyrate in energy-depleted germ-free mouse colon cells (Donohoe et al., 2011), further supporting the concept of butyrate increasing energy supply in epithelial cells. In our study, butyrate increased mRNA expression of acyl-CoA dehydrogenase 9, cytochrome B and ATP synthase, involved in complexes 1, 3 and 5 of the electron transport chain, respectively. The increased mRNA expression shows butyrate induces a genomic shift towards increased oxidative phosphorylation in rumen epithelium.

Coupled with decreased gene expression of lipogenesis genes such as ATP citrate lyase and mitochondrial CoA transporter, our study shows butyrate genomically shifts epithelial metabolism towards energy mobilization in rumen epithelia in SARA cows, in agreement with our hypothesis. Previous studies have highlighted the role of epithelial energy mobilization in preserving barrier integrity and our results show butyrate induces this mobilization, thus may be involved in maintenance of barrier integrity at the genome level during SARA.

\section{CONCLUSION}

In this study, we found exogenous butyrate modulates epithelial expression of genes involved in glycolysis, oxidative phosphorylation and lipogenesis. Genomically, glycolysis and oxidative phosphorylation appear to be upregulated while lipogenesis is largely downregulated. Overall, the changes in gene expression found in this study indicate butyrate induces a genomic shift towards energy production. As energy production is vital for maintaining proper barrier function, butyrate may be beneficial for barrier function in cows experiencing SARA.

While this study found genomic shifts, the corresponding protein abundance and activity was not studied. In the future, a comprehensive study evaluating mRNA abundance, protein abundance and protein activity would add greatly to our understanding of butyrate's mode of action in the rumen. Further, as this study lasted for 7 days only, we could only focus on the short-term effects of butyrate on gene expression. While short-term effects are certainly useful in studying adaptation to high-grain diets, the long-term effects were outside of this study's scope and could be an appropriate focus of future studies.

\section{ACKNOWLEDGEMENT}

The researchers would like to acknowledge funding from the Natural Science and Engineering Research Council of Canada and the Ontario Ministry of 
Agriculture, Food and Rural Affairs. Further, the Ontario Ministry of Agriculture, Food and Rural Affairs' High Quality Personnel Graduate Scholarship to Anne Laarman is acknowledged. Lastly, We would like to thank the staff at University of Guelph Ponsonby Dairy Research Station for their technical assistance and Ivan Girard of Probiotech Inc., for his donation of Proformix butyrate to this study.

\section{REFERENCES}

AlZahal, O., B. Rustomo, N. Odongo, T. Duffield and B. McBride, 2007. Technical note: A system for continuous recording of ruminal $\mathrm{pH}$ in cattle. J. Anim. Sci., 85: 213-217. PMID: 17179558

Aschenbach, J.R., R. Oswald and G. Gabel, 2000. Transport, catabolism and release of histamine in the ruminal epithelium of sheep. Pflugers Arch., 440: 171-178. PMID: 10864012

Aschenbach, J.R., S. Bilk, G. Tadesse, F. Stumpff and G. Gäbel, 2009. Bicarbonate-dependent and bicarbonate-independent mechanisms contribute to nondiffusive uptake of acetate in the ruminal epithelium of sheep. Am. J. Physiol. Gastr. L., 296: G1098- G1098. PMID: 19264953

Baldwin, R.L., S. Wu, W. Li, C. Li and B.J. Bequette et al., 2012. Quantification of transcriptome responses of the rumen epithelium to butyrate infusion using RNA-seq technology. Gene Regul. Syst. Biol., 6: 67-80. PMID: 22654504

Bergman, E.N., 1990. Energy contributions of volatile fatty acids from the gastrointestinal tract in various species. Physiol. Rev., 70: 567-590. PMID: 2181501

Bongard, R.D., K. Yan, R.G. Hoffmann, S.H. Audi and X. Zhang et al., 2013. Depleted energy charge and increased pulmonary endothelial permeability induced by mitochondrial complex I inhibition are mitigated by coenzyme Q1 in the isolated perfused rat lung. Free Radical Biol. Med., PMID: 23912160

Casey, J.R., S. Grinstein and J. Orlowski, 2010. Sensors and regulators of intracellular $\mathrm{pH}$. Nat. Rev. Mol. Cell Biol., 11: 50-61. PMID: 19997129

Davie, J.R., 2003. Inhibition of histone deacetylase activity by butyrate. J. Nutr., 133: 2485S-2493S. PMID: 12840228

Dickman, K.G., S.J. Hempson, J. Anderson, S. Lippe and L. Zhao et al., 2000. Rotavirus alters paracellular permeability and energy metabolism in Caco-2 cells. Am. J. Physiol. Gastrointest. Liver Physiol., 279: G757-G766. PMID: 11005763
Dionissopoulos, L., A.H. Laarman, O. AlZahal, S.L. Greenwood and M.A. Steele et al., 2013. Butyratemediated genomic changes involved in non-specific host defenses, matrix remodeling and the immune response in the rumen epithelium of cows afflicted with subacute ruminal acidosis. Am. J. Anim. Vet. Sci., 8: 8-27. DOI: 10.3844/ajavsp.2013.8.27

Donohoe, D.R., N. Garge, X. Zhang, W. Sun and T.M. O'Connell et al., 2011. The microbiome and butyrate regulate energy metabolism and autophagy in the mammalian colon. Cell Metab., 13: 517-526. PMID: 21531334

Egler, V., S. Korur, M. Failly, J.L. Boulay and R. Imber et al., 2008. Histone deacetylase inhibition and blockade of the glycolytic pathway synergistically induce glioblastoma cell death. Clin. Cancer Res., 14: 3132-3140. PMID: 18483381

Gao, Z., J. Yin, J. Zhang, R.E. Ward and R.J. Martin et al., 2009. Butyrate improves insulin sensitivity and increases energy expenditure in mice. Diabetes, 58: 1509-1517. PMID: 19366864

Graham, C. and N.L. Simmons, 2005. Functional organization of the bovine rumen epithelium. Am. J. Physiol. Regul. Integr. Comp. Physiol., 288: R173181. PMID: 15319221

Krause, K.M. and G.R. Oetzel, 2006. Understanding and preventing subacute ruminal acidosis in dairy herds: A review. Anim. Feed Sci. Technol., 126: 215-236. DOI: 10.1016/j.anifeedsci.2005.08.004

Leonel, A.J. and J.I. Alvarez-Leite, 2012. Butyrate: Implications for intestinal function. Curr. Opin. Clin. Nutr. Metab. Care, 15: 474-479. PMID: 22797568

Lewis, K. and D.M. McKay, 2009. Metabolic stress evokes decreases in epithelial barrier function. Ann. N. Y. Acad. Sci., 1165: 327-337. DOI: 10.1111/j.1749-6632.2009.04036.x

McBrian, M.A., I.S. Behbahan, R. Ferrari, T. Su and T.W. Huang et al., 2013. Histone acetylation regulates intracellular $\mathrm{pH}$. Mol. Cell, 49: 310-321. PMID: 23201122

McBride, B.W. and J.M. Kelly, 1990. Energy cost of absorption and metabolism in the ruminant gastrointestinal tract and liver: A review. J. Anim. Sci., 68: 2997-3010. PMID: 2170320

Mirouse, V., L.L. Swick, N. Kazgan, D. St Johnston and J.E. Brenman, 2007. LKB1 and AMPK maintain epithelial cell polarity under energetic stress. J. Cell Biol., 177: 387-392. PMID: 17470638 
Muneer, A., P.M.S. Alikunju, A. Szlachetka, C. Murrin and J. Haorah, 2011. Impairment of brain endothelial glucose transporter by methamphetamine causes blood-brain barrier dysfunction. Mol. Neurodegenerat., 6: 1-13. PMID: 21426580

Mutsvangwa, T., J. Walton, J. Plaizier, T. Duffield and R. Bagg et al., 2002. Effects of a monensin controlled-release capsule or premix on attenuation of subacute ruminal acidosis in dairy cows. J. Dairy Sci., 85: 3454-3461. PMID: 12512618

Peng, L., Z. He, W. Chen, I.R. Holzman and J. Lin, 2007. Effects of butyrate on intestinal barrier function in a Caco-2 cell monolayer model of intestinal barrier. Pediatric Res., 61: 37-41. PMID: 17211138

Peng, L., Z.R. Li, R.S. Green, I.R. Holzman and J. Lin, 2009. Butyrate enhances the intestinal barrier by facilitating tight junction assembly via activation of AMP-activated protein kinase in Caco-2 cell monolayers. J. Nutr., 139: 1619-1625. PMID: 19625695

Pfaffl, M.W., 2001. A new mathematical model for relative quantification in real-time RT-PCR. Nucl. Acids Res., 29: e45-e45. PMID: 11328886

Plaizier, J.C., D.O. Krause, G.N. Gozho and B.W. McBride, 2008. Subacute ruminal acidosis in dairy cows: The physiological causes, incidence and consequences. Vet. J., 176: 21-31. PMID: 18329918
Ploger, S., F. Stumpff, G.B. Penner, J.D. Schulzke and G. Gabel et al., 2012. Microbial butyrate and its role for barrier function in the gastrointestinal tract. Ann. N.Y. Acad. Sci., 1258: 52-59. PMID: 22731715

Sharma, N., R. Shikhanovich, R. Schloss and M. Yarmush, 2006. Sodium butyrate-treated embryonic stem cells yield hepatocyte-like cells expressing a glycolytic phenotype. Biotechnol. Bioeng., 94: 1053-1063. PMID: 16604521

Spreeuwenberg, M.A.M., J.M.A.J. Verdonk, H.R. Gaskins and M.W.A. Verstegen, 2001. Small intestine epithelial barrier function is compromised in pigs with low feed intake at weaning. J. Nutr., 131: 1520-1527. PMID: 11340110

Williamson, D.H., J. Mellanby and H.A. Krebs, 1962. Enzymic determination of $d(-)-\beta$-hydroxybutyric acid and acetoacetic acid in blood. Biochemical J., 82: 90-90. PMID: 14007241

Xue, Y., S.F. Liao, K.W. Son, S.L. Greenwood and B.W. McBride et al., 2010. Metabolic acidosis in sheep alters expression of renal and skeletal muscle amino acid enzymes and transporters. J. Anim. Sci., 88: 707-717. PMID: 19820050

Zebeli, Q. and B.U. Metzler-Zebeli, 2012. Interplay between rumen digestive disorders and diet-induced inflammation in dairy cattle. Res. Vet. Sci., 93: 1099-1108. DOI: 10.1016/j.rvsc.2012.02.004 